\title{
Ocorrência sazonal e abundância relativa de Tabanidae (Diptera) no Campus Universitário, Manaus, Amazonas.
}

\author{
J. A. Rafael (')
}

\section{Resumo}

Quatro armadilhas de Malaise e uma com isca animal foram utilizadas para a captura de Tabanjdae durante agosto/78 a agosto/79. Mais de trinta espécies foram coletadas, representadas pelos seguintes gêneros: Acanthocera Macq., Catachlorops Lutz, Chlorotabanus Lutz, Chrysops Meig., Cryptotylus Lutz, Diachlorus O.S., Dichelacera Macq., Fidena WIk., Lepiselaga Macq., Poeciloderas Lutz, Phaeotabanus Lutz, Selasoma Macq., Stenotabanus Lutz, Stypommisa End. e Tabanus Linn., este último sendo representado pelo maior número de espécies. O período de atividade e abundância relativa é dado para as espécies mais numerosas, enquanto que para aquelas que ocorreram em menor número é fornecido apenas o registro da ocorrência sazonal.

\section{INTRODUÇÃo}

Vários pesquisadores investigaram a variação de Tabanidae em regiões de clima temperado (Davies, 1959; Pechumann \& Burton, 1969; Thomas, 1970; Roberts, 1971) porém, pouco foi observado nas regiöes tropicais e principalmente na Amazônia. A maioria dos tabanídeos, tanto em regiões temperadas quanto em tropicais é univoltina (Fairchild, 1942) sendo que algumas espécies apresentam estação de vôo muito curta na época chuvosa ou seca, enquanto outras apresentam uma estação de vôo muito longa. Algumas espécies parecem completar mais de um ciclo de vida, durante um ano e outras apresentam ciclo irregular em face da variação no tempo de vida larval, ocorrendo assim, emergência de adultos durante todo o ano.

O presente trabalho é uma complementação do anterior (Rafael \& Charlwood, 1980). Neste, é registrada a ocorrência sazonal dos tabanídeos com menor densidade no Campus Universitário da Universidade do Amazonas, Manăus, Campus escolhido por conter locais alagados e propícios para a oviposição e desenvolvimento dos imaturos de Tabanidae.

\section{MATERIAIS E MÉTODOS}

As capturas foram realizadas de agosto/78 a agosto/79 com quatro armadilhas de Malaise (Townes, 1962) brancas e uma com isca eqüina modificada de Roberts (1977). As armadilhas de Malaise funcionaram ininterruptamente, sendo feita a retirada semanal dos insetos e a captura com isca eqüina realizada duas vezes por mês, coletando todas as mutucas que chegavam para obter 0 repasto sangüineo. Os pormenores das armadilhas, local de coleta e metodologia são dados em Rafael \& Charlwood (1980).

Os dados de precipitação foram tomados junto ao serviço de meteorolgia do INPA, situado aproximadamente a 1.500 metros do local de coleta.

\section{RESUltados E DISCUSSÃo}

Durante o período de coleta, trinta espécies de Tabanidae foram capturadas em ambos os tipos de armadilhas. As espécies assinaladas são mostradas na Tabela I. O gênero Tabanus foi o mais representativo, com onze espécies, apresentando uma delas três variedades e ainda duas espécies não determinadas a nível específico. As espécies mais abundantes no local foram Tabanus dorsiger var. dorsovittatus, $T$. d. var. modestus, T. importunus e Phaeotabanus cajennensis; a variação sazonal, periodicidade diurna e idade fisiológica para estas espécies são dadas em Rafael \& Charlwood (1980).

(1) - Instituto Nacional de Pesquisas da Amazônia, Manaus. 
TABELA 1 - Espécies de Tabanidae capturadas no Campus Universitário, Manaus, durante os meses de agosto/78 a agosto/79

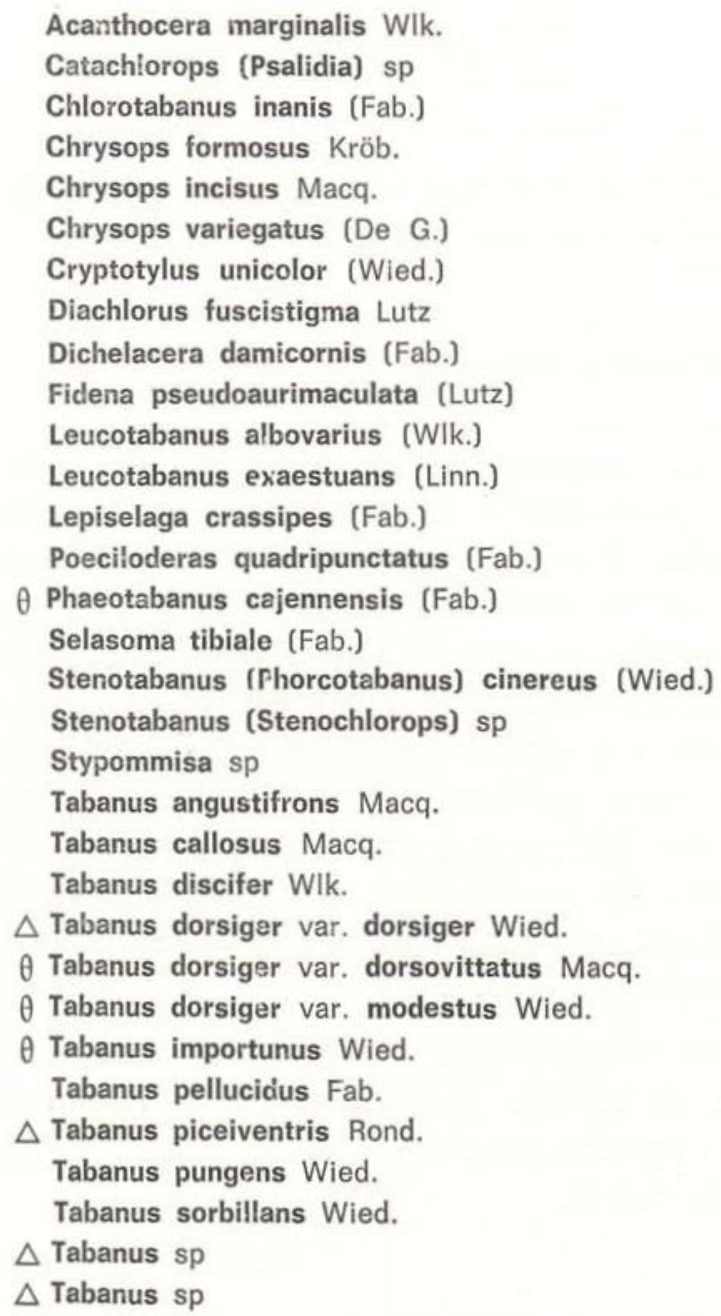

$\theta-$ Os resultados com estas espécies sâo apresentados em Rofoel \& Charlwood (1980).

$\Delta$ - Distribuiçáo sazonal nõo opresentadas neste trabalho.

Para as outras espécies capturadas, a distribuição sazonal e abundância relativa são mostradas na fig. 1, onde se vêem também os dados de precipitação e os respectivos valores de $r$ na correlação de cada espécie com a precipitação. Cada unidade da escala na margem direita indica cinco espécimens e a linha contínua revela apenas um exemplar capturado.

Das espéeies relacionadas, Chlorotabanus inanis, Chrysops incisus e C. formosus apresentaram correlação negativa $(r=-0,48,-0.52$ e $-0,48$ respectivamente) com a precipitação a nivel de $1 \%$, enquanto Tabanus discifer e Selasoma tibiale apresentaram correlação positiva $(r=0,60$ e 0,42$)$ a nível de $0,5 \%$ e $1 \%$ respectivamente. As outras espécies não apresentaram qualquer correlação significativa e este resultado explica-se provavelmente em face do baixo número capturado no local, não implicando, no entanto, que estas espécies não estejam correlacionadas com a precipitação. Quando forem realizadas capturas em outros locais, onde estas espécies se mostrem mais abundantes, poder-se-á revelar uma correlação positiva ou negativa em relação à precipitação.

Pela distribuição sazonal, nota-se que há espécies com estação de vôo na época seca (Chrysops incisus) ou chuvosa (Tabanus discifer) e ainda espécies com estação de vôo curta (Tabanus pungens) ou longa (Chlorotabanus inanis). Poucas espécies mostraram evidências de mais de uma geração por ano ou de voarem através do ano em igual abundância.

As larvas de tabanídeos encontram-se em uma grande variedade de habitats. A maioria das espécies é aquática ou semi-aquática, vivendo em águas paradas ou correntes, águas contidas em buracos de árvores ou nas bainhas das folhas de Bromeliaceae (Fairchild, 1981). Assim sendo, a longa estação de vôo de algumas espécies pode estar relacionada à constante emergência de adultos, dada a circunstância do local alagado e propício para o desenvolvimento dos imaturos de tabanídeos na área em que foi realizada a coleta.

Quanto às estações climáticas, apesar da possibilidade de atividade durante 0 ano inteiro nos trópicos, a maioria das espécies apresentam uma estação definida de vôo e isto provavelmente seja uma estratégia para evitar competições interespecíficas no mesmo hospedeiro, já que a hipótese de ocorrer hospedeiros diferentes em diferentes épocas é improvável nesta região. Também pode estar relacionado à estratégia de evitar competições interespecíficas o hábito de certas espécies picarem regiões diferentes do hospedeiro, como Leucotabanus exaestuans que pousa exclusivamente nas proximidades da orelha do ca- 
valo, Tabanus dorsiger nas patas, Chrysops na cabeça, Selasoma na barriga. Tais hábitos já foram observados por outros autores, como Bouvier (1952), Page (1972) e Hollander \& Wright (1980).

A densidade de tabanídeos numa determinada região do animal parece não limitar o número de pousos adicionais. As mutucas que já estavam pousadas ou mesmo alimentandose não foram perturbadas por outras mutucas que pousavam. O desalojamento ocorria quando o animal reagia, abanando a cauda ou batendo as patas ou contraindo os músculos.

Ainda para as espécies com menos de dez exemplares coletados, é dado apenas o registro da ocorrência sazonal na fig. 2. Algumas destas espécies são muito abundantes em outras áreas da região Amazônica (observação pessoal) e, portanto, nada podemos concluir sobre a ocorrência sazonal destas espécies. Contudo, apesar do baixo número coletado, os registros tendem a refletir o período de maior atividade de vôo de uma determinada espécie. Para as espécies que normalmente ocorrem com baixa densidade e por curtos períodos, é necessário que se façam capturas com animais ou mesmo armadilhas, em diferentes habitats, propiciando assim uma determinação segura da ocorrência sazonal das espécies menos populosas.

$O$ indice de diversidade $(\alpha=3,36$ onde $S=30$ ) calculado através da fórmula de Margalef (apud Southwood, 1971), foi relativamentє baixo, considerando-se que as coletas foram realizadas na região tropical, onde a diversidade é característica da mesma, e que os tabanídeos formam um grupo grande, com quase mil espécies na região Neotropical (Fairchild, 1971). A baixa diversidade, com predominância de uma espécie, $85 \%$ de Tabanus dorsiger, se deu provavelmente porque as coletas foram realizadas numa área aberta e perturbada (ca-

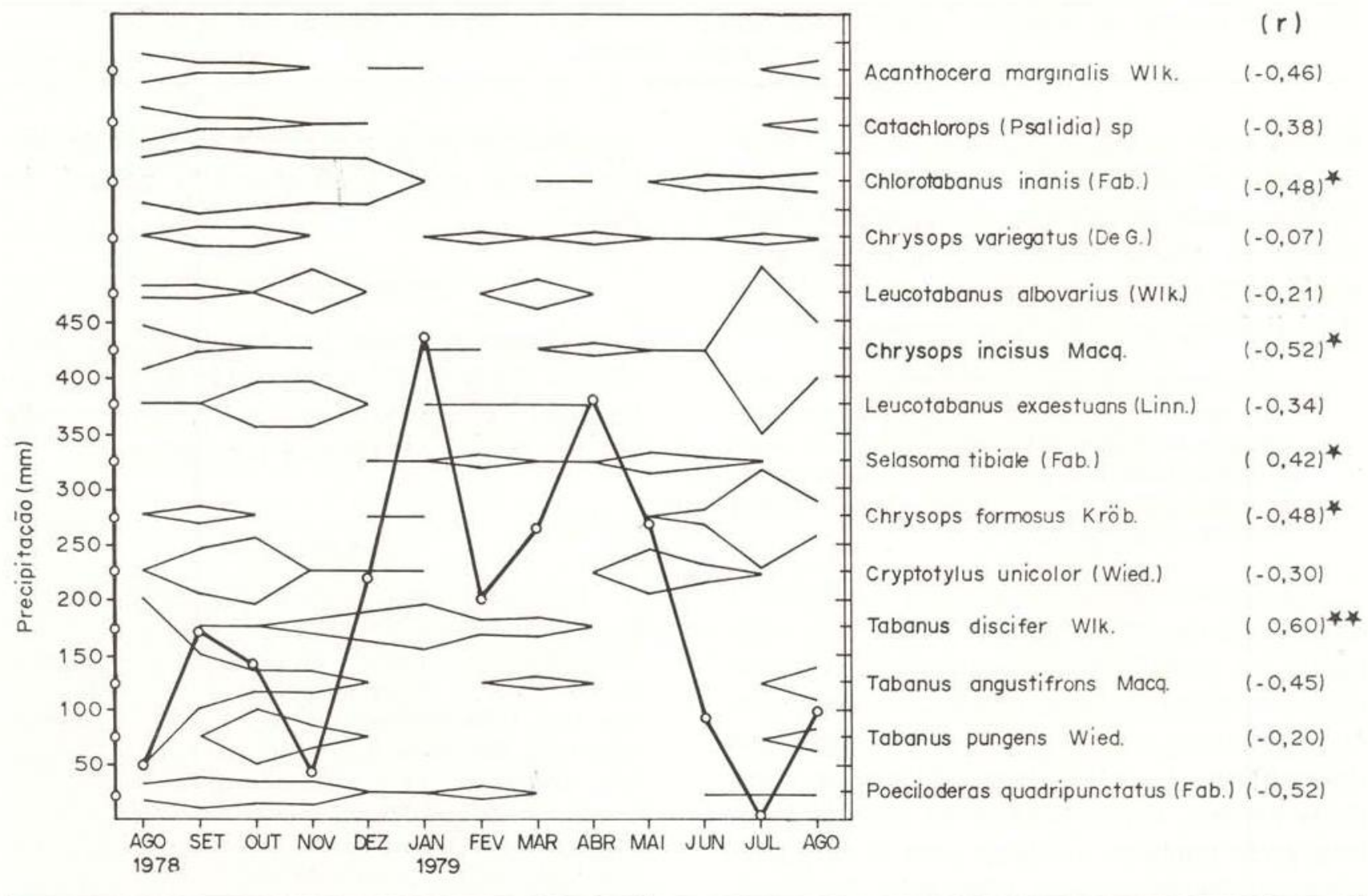

Fig. 7 - Distribuiçăo sazonal e abundância relativa de Tabanidae capturados no Campus Universitário, Manaus e seus respectivos valores de $\mathbf{r}$ na correlação com precipitação.

$$
\mathrm{P}<0,1 ; 11 \mathrm{~g} . \mathrm{l} \text {. } \quad \mathrm{P}<0,05 ; 11 \mathrm{~g} . \mathrm{l} \text {. }
$$




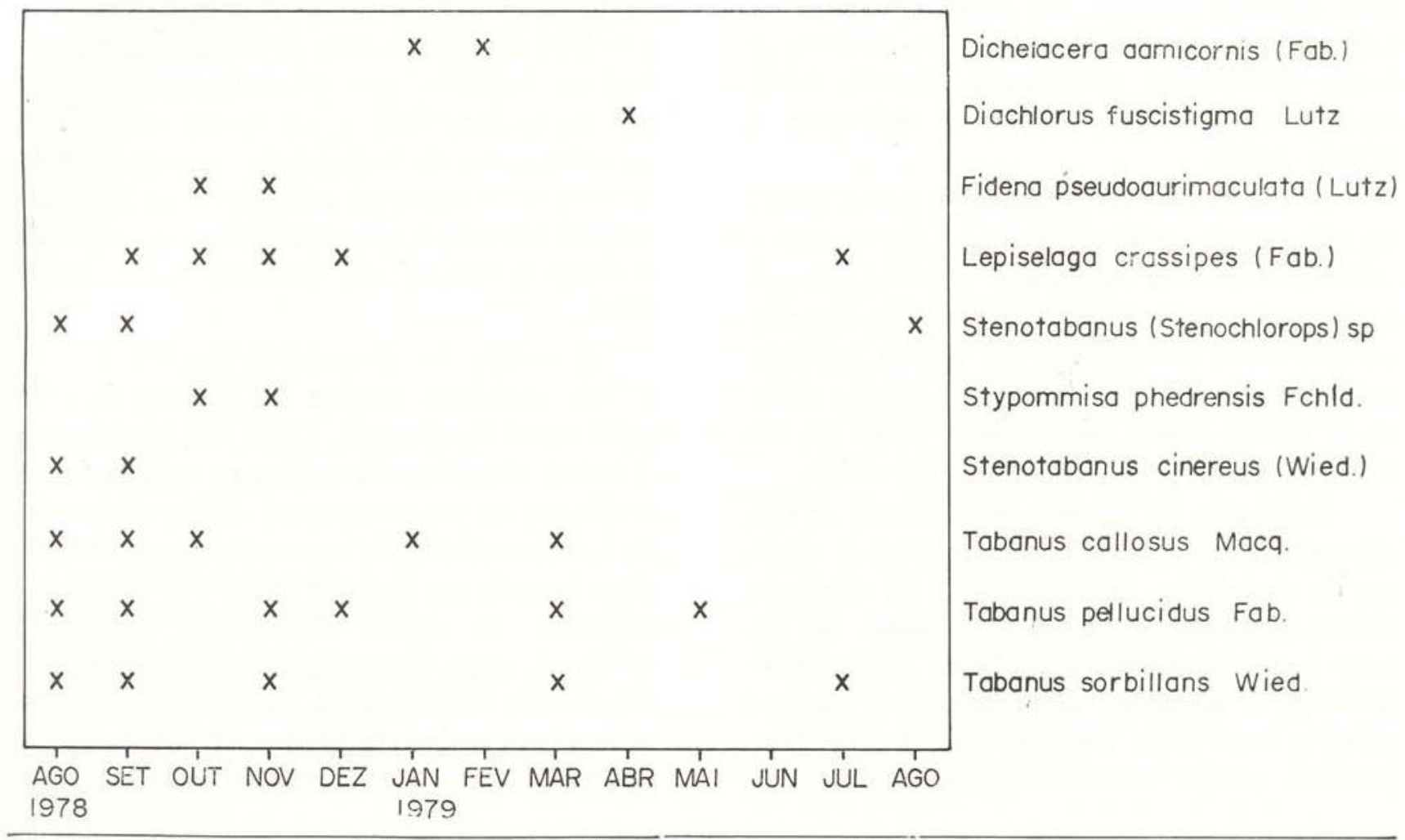

Fig. 2 - Registro da ocorrência sazonal de Tabanidae, com menos de dez especimens capturadas no Campos Universitário, Manaus.

poeira) onde existe a possibildade de a espécie mais abundante estar dominando. As variedades mais abundantes da espécie dorsiger foram: $d$. dorsovittatus e $d$. modestus, ocorrendo com menos freqüência $d$. dorsiger. Fairchild (inform. carta) diz que estas variedades:

...they are not, of course, subspecies in the ordinary sense, but seem to be somehow environmentally correlated, the modestus form in areas of highest rainfall or in forest habitats, the others in more open country. Dorsiger dorsiger seems dominant in S. Brazil.

O relacionamento ambiental, conforme a informação de Fairchild, confirma-se pela coloração das variedades deste complexo; a variedade modestus é a mais escura, habitando preferencialmente a floresta primária; a variedade dorsovittatus é a mais clara e foi a mais abundante na área aberta em que foi relizada a coleta, onde também o habitat para o desenvolvimento das larvas era altamente favorável, (Goodwin \& Murdoch, 1974), pois o local continha capins sobre o solo alagadiço; a variedade dorsiger, intermediária em coloração, ocorreu com poucos espécimens, sendo que esta forma aparece mais freqüentemente no sul do país.

\section{Agradecimentos}

O autor agradece aos doutores G.B. Fairchild e Paulo F. Buhrnheim pela leitura e críticas ao manuscrito e ao Dr. W. Benson pela orientação nos cálculos de estatística.

\section{SUMMARY}

Four Malaise trap and one bait animal were utilized for the capture of Tabanidae from August/78 until August/79. Thirty species were collected, represented by the following genera: Acanthocera Macq., Catachlorops Lutz, Chlorotabanus Lutz, Chrysops Meig., Cryptobylus Lutz, Diachlorus O.S., Dichelacera Macq., Fidena Wlk., Lepiselaga Mecq., Poeciloderas Lutz, Phaeotabanus Lutz, Selasoma Macq., Stenotabanus Lutz, Stypommisa End. and Tabanus Linn., this last genus being represented by the largest number of species. The period of activity and relative abundance is given for the most abundant species, while that for those that occur in fewer number oniy a register of seasonal occurence is furnished. 


\section{REFERENCIAS BIBLIOGRAFICAS}

BOUVIER, G.

1952 - Notes sur les tabanidés de la région de Campinas (Estado S. Paulo) Brésil. Mem. Inst. Oswaldo Cruz, 50: 581-596.

DAVIES, D.M.

1959 - Seasonal variation of tabanids (Diptera) in Algonquin Park. Ontario. Can. Entomol., 91: 548-553.

FAIRCHILD, G.B.

1942 - The seasonal distribution of some Tabanidae (Diptera) in Panama. Ann. Ent. Soc. Am. 35: 85-91.

1971 - Family Tabanidae, fasc. 28: 1-263. In: N. Papavero (Ed.), A catalogue of the Diptera of the United States. Museu de Zoologia, Univ. São Paulo.

1981 - Tabanidae, pp. 290-301. In: Aquatic Biota of Tropical South America Part I. Arthropoda. Hurlbert, Rodriguez e Santos (Ed.).

GOODWIN, J.T. \& MURDOCH, W.P.

1974 - A study of some immature Neotropical Tabanidae. Ann. Ent. Soc. Am., 67 (1): 85-113.

HIOLLANDER, A.L. \& WRIGHT, R.E.

1980 - Impact of tabanids on cattle: blood meal size and preferred feeding sites. J. Econ. Entomol., 73: 431-433.

PAGE, W.A.

1972 - Feeding behaviour and trypanosomatid infections of some tabanids and Culicidae in Colombia. J. Ent. (A), 47 (1): 1-13.
FECHUMANN, L.L. \& BURTON, J.J.S.

1969 - Seasonal distribution of Tabanidae (Diptera) at Texas Hollow, New York in 1968 Mosq. News, 29 (2): 216-220.

RAFAEL, J.A. \& CHARLWOOD, J.D.

1980 - Idade fisiológica, variação sazonal e periodicidade diurna de quatro populaçōes de Tabanidae (Diptera) no Campus Universitário, Manaus, Brasil. Acta Amazonica, 10 (4): 907-927.

R.OBERTS, R.H.

1971 - The seasonal appearance of Tabanidae as determined by Malaise trap collections. Mosq. News, 31 (4): 509-512.

1977 - A technique for the collection of engorged Tabanidae. Mosq News, 37 (4): 763-764

SOUTHWOOD, T.R.E.

1971 - Ecological Methods. Chapman and Hall Ltda, $391 \mathrm{pp}$.

THOMAS, A.W.

1970 - Seasonal occurence and relative abundance of Tabanidae (Diptera) in three localities in Alberta. Quaest. Ent., 6: 293-301.

TOWNES, $\mathrm{H}$.

1962 - Design for a Malaise trap Proc. Ent. Soc. Wash., 64 (4): 253-262.

(Aceito para publicação em 16/11/81) 